\title{
Pure squamous cell carcinomas of gall bladder: A series of 4 cases
}

\author{
Suhail Farooq 1,", Ambreen Beigh ${ }^{2}$, Sheikh Junaid ${ }^{3}$, Baba Iqbal Khaliq ${ }^{4}, J_{i b r a n ~ A m i n}{ }^{5}$ \\ $\mathbf{1 , 2 , 4 , 5}$ Demonstrator, ${ }^{3}$ Senior Resident, ${ }^{\mathbf{1 , 2}, \mathbf{4}, \mathbf{5}}$ Dept. of Pathology, ${ }^{3}$ Dept. of Surgery, Government Medical College, Jammu and
} Kashmir, India

*Corresponding Author: Suhail Farooq

Email: dsfb1983@gmail.com

Received: $28^{\text {th }}$ April, 2018

Accepted: $14^{\text {th }}$ June, 2018

\begin{abstract}
Introduction: Pure squamous cell carcinoma is an exceptional malignancy with a few case series available in literature. The overall clinical and pathological picture is highly variable and prognosis dismal. Our study describes a series of 4 patients who presented with squamous cell carcinoma of the gallbladder along with their clinico-pathological characteristics.

Materials and Methods: The clinical presentation, laboratory investigations, radiological details were retrieved from the hospital records. The gross features and histopathological findings were collected from the departmental archival material and were analyzed by a single pathologist.

Result: The clinical and pathological picture of the patients with pure squamous cell carcinoma was highly variable. The radiological features varied from a diffuse thickening of gall bladder wall, a exophytic intraluminal mass in the gall bladder to a large homogenous irregular mass in the right hypochondrium on ultrasonography. The gross features of gall bladder specimens varied from a diffuse infiltrating growth, a polypoid mass arising from the wall, to a large highly friable mass in right hypochondrium infiltrating into adjacent colon. The microscopic features varied from a tumor composed of groups, islands or nests of atypical squamous cells with dyskeratosis and keratin pearl formation to a tumor comprising of cells arranged in solid sheets with areas of comedo necrosis and keratin pearl formation. The prognosis was dismal.

Conclusion: The clinical features and overall survival of patients with pure squamous cell carcinoma is more or less similar to the more common adenocarcinoma of gallbladder, however, the no. of cases studied is small to draw conclusions indicating the need for more extensive study.
\end{abstract}

Keywords: Pure squamous cell carcinoma, Gall bladder mass, Adenocarcinoma gall bladder, Intraluminal mass gall bladder.

\section{Introduction}

Carcinoma of the gall bladder usually presents after $5^{\text {th }}$ decade of life and is more commonly seen in the females. It is the most common malignancy of the extra-hepatic biliary tract.1The gross presentation may vary from a diffusely infiltrating growth to a polypoid intraluminal mass. ${ }^{2}$ The most common histopathological type of carcinoma of the gallbladder is Adenocarcima. ${ }^{3}$ The clinical onset is insidious and the signs and symptoms mimic those of chronic cholecystitis with cholelethiasis. The most common clinical findings are the upper abdominal pain, jaundice, anorexia, nausea and vomiting.

Pure squamous cell carcinoma of the gallbladder is a rare malignancy mostly described in literature as individual case reports. ${ }^{4,5}$ The clinical presentation and overall survival of patients presenting with squamous cell carcinoma of the gallbladder is highly variable. Our study describes a series of 4 patients who presented with pure squamous cell carcinoma of the gallbladder along with their clinico-pathological characteristics.

\section{Materials and Methods}

Four cases based on the histopathological examination of the post operative specimens received by the Postgraduate Department of Pathology, Government Medical College, Srinagar were included in the study. The clinical presentation, laboratory investigations, radiological details, pre-operative diagnosis and intra-operative findings, were retrieved from the hospital records. The gross features and histopathological findings were collected from the departmental archival material and were analyzed by a single pathologist and the clinico-pathological characteristics of the cases described. The four cases included in this study were identified as pure squamous cell carcinomas on histopathology with no invasive glandular component on microscopic examination.

\section{Results and Observations}

The present study included 1 male and 3 females. The age of the patients included in our study was 53, 67,70 and 75 . The clinical picture of the patients showed considerable variation. One of the patients a $75 \mathrm{yr}$ old male ex-smoker presented with multiple episodes of vomiting, epigastric discomfort and loss of weight while the other a 70yr elderly female presented with painless swelling in right hypochondrium which had increased in size during a period of 6 months and jaundice. The other two patients were middle aged females who had presented with pain and discomfort in right hypochondrium. On palpation during the examination, the elderly female presented with a palpable mass in the right hypochondrium. The other 
three cases discerned no significant finding on examination. There was no abnormal finding on Routine Hemogram in three patients while the forth patient the elderly female presented with hemoglobin 8 $\mathrm{g} / \mathrm{dL}$. The liver function tests showed no abnormal findings in any of the patients.

The radiological features varied from a diffusely thickened wall of gall bladder, a rounded intraluminal mass $3 \times 2 \mathrm{~cm}$ arising from the wall of gall bladder (Fig. 1), to a large homogenous echo texture mass measuring $12 \times 10 \mathrm{~cm}$ in the right upper abdomen on ultrasonography. CECT abdomen of the two patients in whom available showed, a solid intraluminal polypoid enhancing mass measuring $30 \times 20 \mathrm{~mm}$ in posterior wall of gall bladder (Fig. 2), to a large enhancing mass measuring $12 \times 10 \mathrm{~cm}$ involving right upper abdomen and abutting portion of liver. A simple cholecystectomy was performed in two patients, cholecystectomy along with lymph node dissection in one and cholecystectomy plus resection of growth, followed by side-side colonocolonic anastomosis, along with lymph node dissection was done in other patient. The postoperative period was uneventful in all these patients. They were discharged within 7 to 10 days after surgery.

Gross examination varied from a diffuse infiltrating growth of the wall of gall bladder, a polypoid intraluminal mass measuring 3.x $2 \mathrm{~cm}$ in the wall of body of gall bladder (Fig. 3), to a large friable mass measuring $10 \times 8 \mathrm{cms}$ involving the whole of the gall bladder and infiltrating into adjacent colon (Fig. 4). Microscopic examination varied from a tumor composed of groups, islands and nests of atypical squamous cells with dyskeratosis and keratin pearl formation (Fig. 5) to a tumor comprising of cells arranged in solid sheets with areas of comedo necrosis with infiltration into muscle layer of gall bladder (Fig. 6). Many dyskeratotic cells were seen with infiltration into adjacent colon through serosal side in one case. One case depicted vascular and perineural invasions.

All of the four cases were carcinomas with advanced stages, i.e. carcinomas with invasion beyond the muscularis. Two out of four cases were pT2 (invasion into perimuscular connective tissue) while one was pT3 (infiltration into adjacent liver) and the other one was pT4 (infiltration into extrahepatic organs). Lymph node metastasis was seen in two cases. During follow-up one of the patient elderly female died within a period of 3 months. The other two patients died within 6 months and the forth one was doing well for a period of one year.

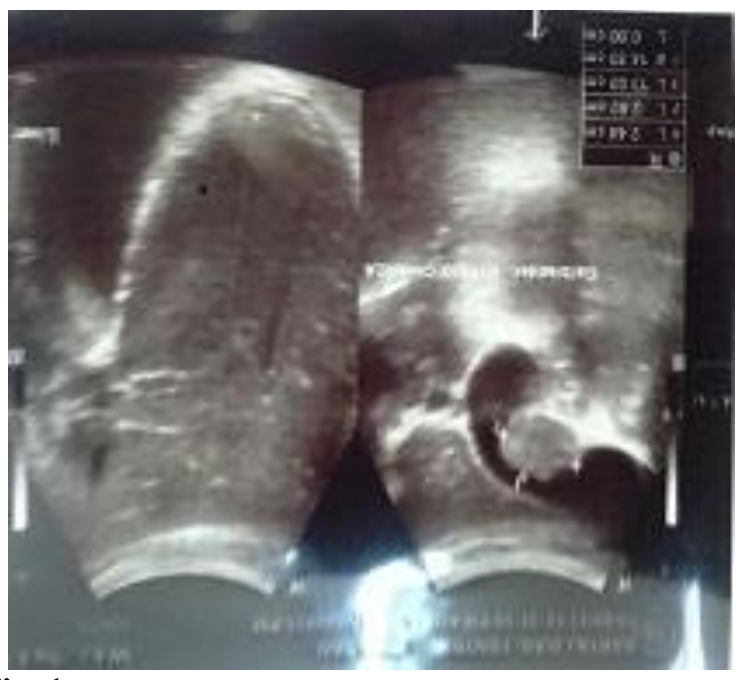

Fig. 1

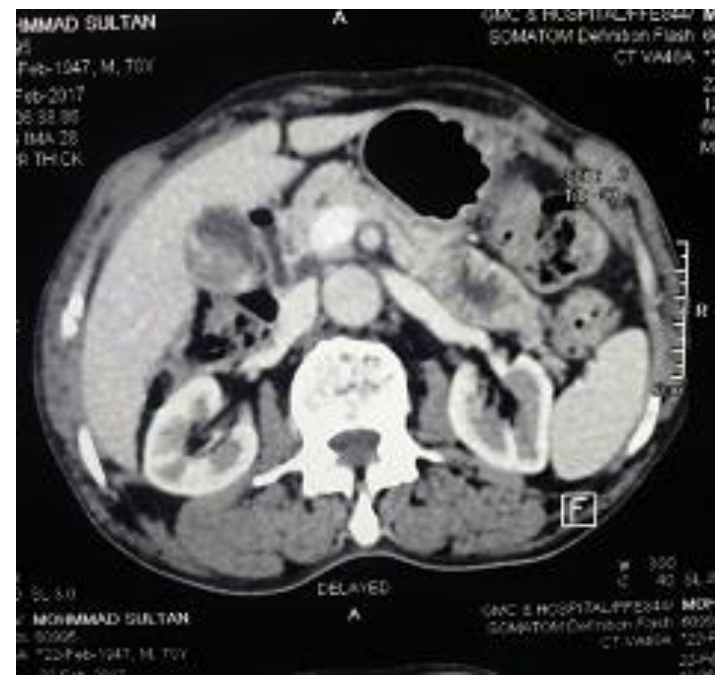

Fig. 2

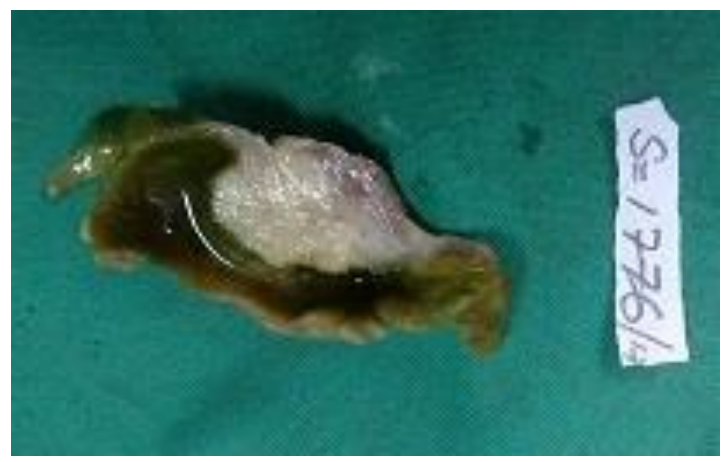

Fig. 3 


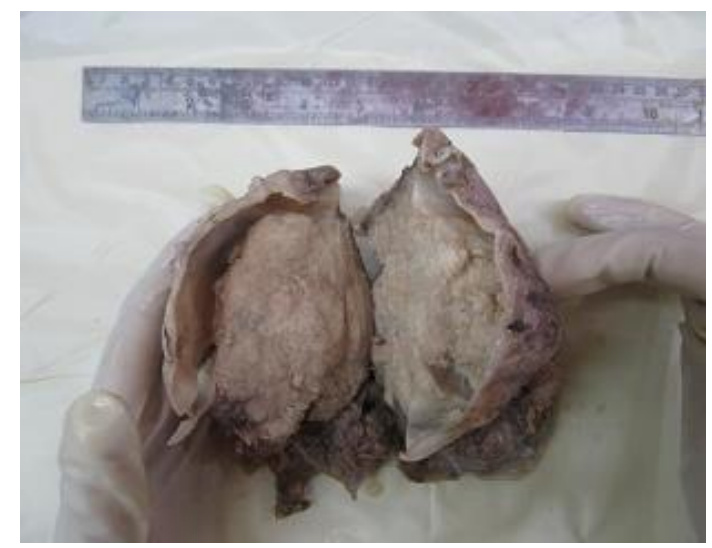

Fig. 4

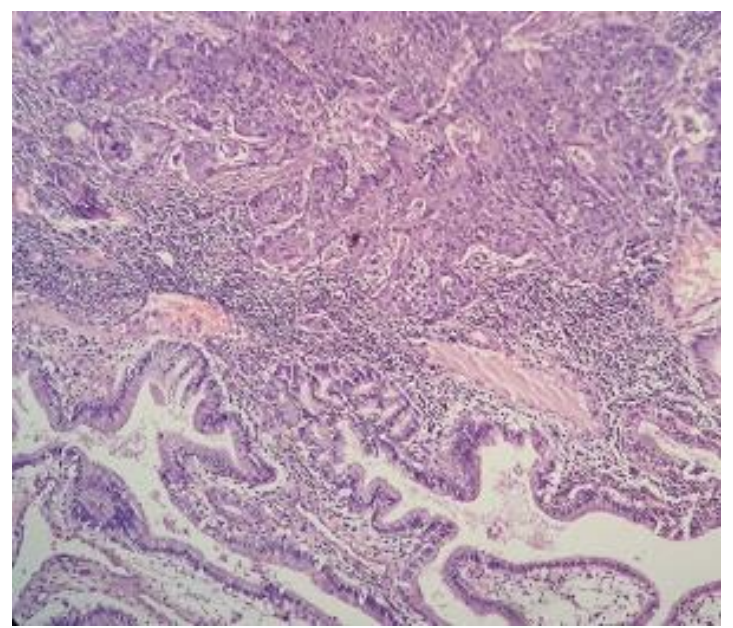

Fig. 5

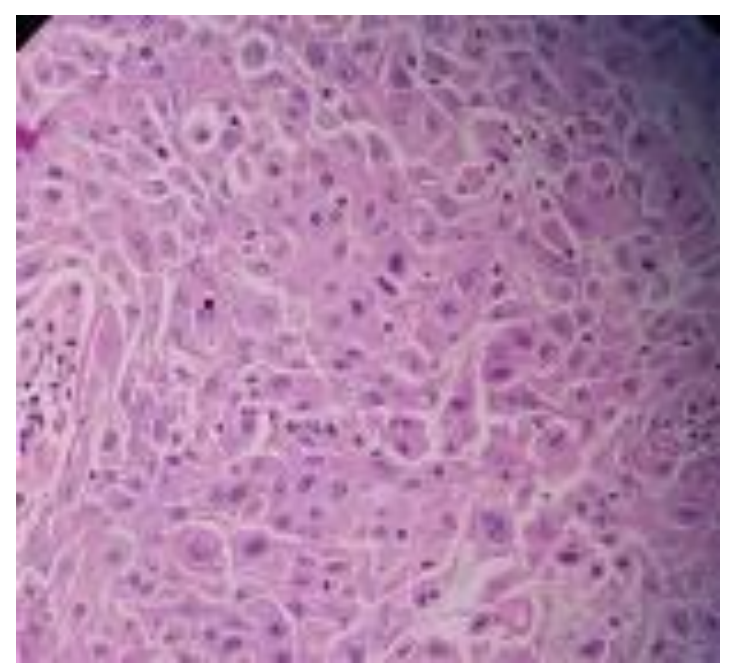

Fig. 6

\section{Discussion}

Pure squamous cell carcinoma of the gall bladder is an uncommon malignancy. The clinical presentation and overall survival of patients presenting with pure squamous cell carcinoma of the gallbladder has not been described thoroughly. In our analysis of 4 cases the age of presentation was found to be above 50 years of age with female preponderance which is comparable with the age of presentation as well as predilection for female sex in case of adenocarcinoma of gall bladder - the most common histopathological type. ${ }^{6}$ The presenting symptoms of the patients in this study were pain right upper abdomen, anorexia, and vomiting. These nonspecific symptoms mimicking acute cholecystitis are the usual clinical presentation of carcinoma of gall bladder - mostly adenocarcinomas. One patient in this analysis presented with a painless mass in right upper abdomen and anemia which has been described as one of the presentations of carcinoma gall bladder - mostly adenocarcinomas. $^{7}$

On ultrasonography the presentation of the 4 cases studied varied from a diffusely thickened wall of gall bladder in two cases to a rounded intraluminal mass in third case (Fig. 1) and a large homogenous echo texture mass measuring 12x10 involving right upper abdomen in the forth case. CECT abdomen of the later two patients in whom it was advised and available revealed, a solid intraluminal polypoid enhancing mass measuring $30 \times 20 \mathrm{~mm}$ in posterior wall of gall bladder in one and a large mass with contrast enhancement measuring $12 \times 10 \mathrm{~cm}$ involving right hypochondrium and abutting the portion of liver in another. Studies have described that the carcinomas of gall bladder usually present as a diffuse thickening of the gall bladder wall in most cases or a polypoid intra-luminal mass or a mass arising from gall bladder involving other adjacent organs mostly liver. Our findings are consistent with these studies. . $^{8-10}$

Grossly carcinomas of the gall bladder, most of them adenocarcinoma present either as a diffusely growing thickened firm wall to a intraluminal polypoid mass. In this study on gross two cases showed a diffuse firm infiltrating growth of gallbladder wall, one case showed a polypoid exophytic mass in the lumen of gall bladder wall; and one showed a large friable mass arising from gall bladder and involving the whole of the right hypochondrium and infiltrating into adjacent colon. Most of the pure squamous cell carcinomas appear to be well differentiated, with prominent keratinization on microscopic examination. Many show abundant keratin pearls, dyskeratotic cells and central deposition of dense keratin material. Comedo like necrosis may be noted in many cases more in the infiltrative nests. ${ }^{11,12}$ In our cases microscopic examination varied from a tumor composed of a tumor composed of groups, islands and nests of atypical squamous cells with dyskeratosis and keratin pearl formation to a tumor comprising of cells arranged in solid sheets with areas of comedo necrosis with infiltration into muscle layer of gall bladder.

Carcinomas of gall bladder generally present with advanced stage. Most of the cases have spread beyond muscularis at the time of presentation. They have a great propensity to spread directly to liver. ${ }^{13}$ The 
frequency of lymph node involvement is highly dependent on the depth of invasion of the primary tumor. ${ }^{14}$ Almost half of the patients already have metastatic disease at the time of surgery. ${ }^{15}$ In our study all the cases were advanced carcinomas, with invasion beyond the muscularis. Two out of four cases were pT2 (invasion into perimuscular connective tissue) while one was pT3 (infiltration into adjacent liver) and the other one was pT4 (infiltration into extrahepatic organs). Lymph node metastasis was seen in two cases. During follow-up one of the patients elderly female died within a period of 6 months. The other two patients died within 8 months and the forth one was doing good for a period of one year. ${ }^{16-18}$

\section{Conclusion}

The clinical presentation and overall survival of patients presenting with squamous cell carcinoma of the gallbladder is highly variable. The presentation may vary from a diffuse thickened wall or an intraluminal mass to infiltrative growth, from a well differentiated to poorly differentiated tumor, from the localized disease with a favorable outcome to an advanced disease with a guarded prognosis. The present study is an attempt in this direction; however, the number of cases in this study is too small to draw conclusions indicating the need for more extensive study.

\section{References}

1. Randi G, Franceschi S, La Vecchia C. Gallbladder cancer worldwide: geographical distribution and risk factors. Int J Cancer. 2006;118:1591-1602.

2. Sons HU, Borchard F, Joel BS: Carcinoma of the gallbladder. Autopsy findings in 287 cases and review of the literature. J Surg Oncol. 1985;28:199-206.

3. Longnecker DS, Terhune PG: The case for parallel classification of biliary tract and pancreatic neoplasms. Mod Pathol. 1996;9:828-837.

4. Roppongi T, Takeyoshi I, Ohwada S, Sato Y, Fujii T, Honma M, et al. Minute squamous cell carcinoma of the gallbladder: a case report. Jpn J Clin Oncol. 2000;30:435. doi: 10.1093/jjco/hyd010. PubMed PMID: 10770570.

5. Karasawa T, Itoh K, Komukai M, Ozawa U, Sakurai I, Shikata T. Squamous cell carcinoma of gallbladder-report of two cases and review of literature. Acta Pathol Jpn. 1981;31:299-308. PubMed PMID: 7257770.

6. Donohue JH, Stewart AK, Menck HR: The National Cancer Data Base report on carcinoma of the gallbladder, 1989-1995. Cancer. 1998;83:2618-2629.
7. Pandey M, Pathak AK, Gautam A, Arya NC, Shukla VK. Carcinoma of gallbladder: a retrospective review of 999 cases. Digest Dis Sci. 2001;46:1145-51.

8. Franquet T, Montes M, Ruiz de Azua Y, Jimenez F J, Cozcolluela $\mathrm{R}$. primary gallbladder carcinoma: imaging findings in 50 patients with pathologic correlation. Gastrointest Radiol. 1991;16:143-148.

9. Li B, Xu XX, Du Y, Yang HF, Li Y, Zhang Q. Computed tomography for assessing resectability of gallbladder carcinoma: a systematic review and meta-analysis. Clin Imaging. 2013;37(2):327-33.

10. Kim JH, Lee JY, Baek JH, Eun HW, Kim YJ, Han JK, et al. High-resolution sonography for distinguishing neoplastic gallbladder polyps and staging gallbladder cancer. AJR Am J Roentgenol. 2015 Feb. 204 (2):W150-9

11. Sons HU, Borchard F, Joel BS: Carcinoma of the gallbladder. Autopsy findings in 287 cases and review of the literature. J Surg Oncol 1985; 28:199-206.

12. Juan C Roa1, Oscar Tapia1, Asli Cakir, Olca Basturk, Nevra Dursun, Deniz Akdemir, Burcu Saka et al. Squamous cell and adenosquamous carcinomas of the gallbladder: clinicopathological analysis of 34 cases identified in 606 carcinomas Modern Pathology. 2011;24:1069-1078.

13. Wakai T, Shirai Y, Sakata J, Nagahashi M, Ajioka Y, Hat akeyama K: Mode of hepatic spread from gallbladder carcinoma: an immunohistochemical analysis of 42 hepatectomized specimens. Am J Surg Pathol. 2010;34:65-74.

14. Tsukada K, Kurosaki I, Uchida K, Shirai Y, Oohashi Y, Yokoyama N, Watanabe H, Hatakeyama K: Lymph node spread from carcinoma of the gallbladder. Cancer. 1997;80:661-667.

15. Hamrick Jr RE, Liner FJ, Hastings PR, Cohn Jr I: Primar y carcinoma of the gallbladder. Ann Surg. 1982;195:270273.

16. Waisberg J, Bromberg SH, Franco MI, et al. Squamous cell carcinoma of the gallbladder. Sao Paulo Med J. 2001;119:43.

17. de Aretxabala X, Roa I, Burgos L, et al. Gallbladder cancer: an analysis of a series of 139 patients with invasion restricted to the subserosal layer. $J$ Gastrointest Surg. 2006;10:186-192.

18. Appelman RM, Morlock CG, Dahlin DC, Adson MA: Lo ng term survival in carcinoma of the gallbladder. Surg Gynecol Obstet. 1963;117:459-464.

How to cite this article: Farooq S, Beigh A, Junaid S, Khaliq B.I, Amin J. Pure squamous cell carcinomas of gall bladder: A series of 4 cases. Indian J Pathol Oncol. 2018;5(4):671674. 